Campos Gil, Isabel.

Artista investigadora; Alumna del Máster de Investigación en Prácticas Artísticas y Visuales.

Universidad de Castilla- La Mancha.

\title{
El lenguaje y los movimientos de ocupación. Un recorrido por las nociones dominantes del lenguaje en relación con la ocupación como movimiento contra-cultural en la hegemonía occidental.
}

\section{Language and encroachment movements. A route through the dominant notions of language in relation with encroachment such movements counter-cultural in occidental hegemony.}

\author{
TIPO DE TRABAJO: \\ Comunicación. \\ PALABRAS CLAVE: \\ Investigación artística; Lenguaje; Discursos; Arte contemporáneo; Arte Conceptual.
}

KEY WORDS:

Artistic research; Language; Speeches; Contemporary art; Conceptual Art.

RESUMEN.

\begin{abstract}
A través del estudio de la perfomatividad, se introduce un planteamiento que parte de una serie desvíos de las estrategias de los movimientos sociales de ocupación para la transformación de los códigos del lenguaje en una relación transfeminista.
\end{abstract}

Desde una mirada pos-crítica de la producción de subjetividades políticas y su constitución en los sujetos, se plantea el lenguaje como una tecnología de identidades de género y en su relación con el orden social hegemónico. Hacia una lectura posestructuralista, estableciendo alegorías sobre la estrategia del ajedrez en la lingüística (desde las metáforas fílmicas y literarias), se plantea el tablero de ajedrez como espacio cuya propiedad se encuentra en la figura paterna y en la que gobierna un régimen tanatopolítico, abandonado por las luchas cotidianas.

Presenta reflexiones poniendo el tablero de ajedrez como espacio entre lo habitado y lo deshabitado, entre la presencia y la ausencia, entre lo abandonado y las reglas. Entendido como sistema binario por su representación, basada en la organización de dos tonalidades antagónicas y presentado los sujetos del juego en oposición que se puede ver como resultado de espejo. A través del análisis del juego, cuya presencia de las mujeres como sujetos históricos, además, ha estado anulada, presenta la dicotomía del pensamiento posmodernista y las estructuras que lo sustentan. Con el objeto y el sujeto puesto en el análisis de estudio, se realiza una mirada que constituye un recorrido por los movimientos sociales de ocupación en cuanto al modelo de pensamiento dominante, poniendo en jaque las nociones de signo de Saussure interpeladas y desequilibradas por la teoría del discurso feminista. Estableciendo en el lenguaje, a su vez, lugares de resistencia política. 


\section{ABSTRACT.}

Through the study of performativity, an approach is introduced that starts from a series of desviations from the strategies of the social movements of occupation for the transformation of the codes of language into a transfeminist relation.

From a post-critical view of the production of political subjectivities and its constitution in subject, language is proposed as a technology of gender identities in relation to the hegemonic social order. Towards a poststructuralist reading and establishing allegories on the strategy of chess in linguistics -from the filmic and literary metaphors-, the chessboard is considered a space whose property is in the paternal figure and in which governs a tanatopolitical regime, abandoned by daily struggles.

This article presents reflections putting the chessboard as a space between the inhabited and the uninhabited, between the presence and absence, between the deserted and the rules. Understanding it as a binary system by its own representation based on the organization of two antagonistic tonalities and on a presentation of the subjects of the game in opposition that can be seen as a result of reflection. Through the analysis of the game, whose presence of women as historical subjects has been annulled, presents the dichotomy of postmodernist thinking and the structures that support it. With the object and the subject placed in the study analysis, it mades a view that constitutes a drift of the social movements of occupation in terms of the dominant thinking model, putting in the check the notions of Saussure's sign interpellated and unbalanced by the theory of feminist discourse. Also, establishing in language some places of political resistance.

\section{CONTENIDO.}

\section{Desde los estudios sobre el lenguaje.}

Interpelación desde el feminismo posestructuralista a la semiótica.

El lenguaje ha tenido siempre una presencia importante como objeto de estudio e interrogante. Cada época o civilización ha contribuido a la carga de significantes dependiendo de las estructuras culturales que la configuraban. Por lo que, siguiendo la línea de investigación de Julia Kristeva, no podemos no abstenernos de afirmar que el lenguaje es reductiblemente un instrumento del pensamiento. Semejante concepción llevaría a pensar que el lenguaje expresa, como herramienta, una idea exterior a él (Kristeva, 1999: 37). Desde esta perspectiva podemos entender el lenguaje como la matriz por la cual se producen y reproducen los espacios, los sujetos y el lugar se coproducen.

¿Cómo podríamos salirnos del lenguaje, como podríamos no jugar sus reglas, como podríamos no seguir el mismo juego? Me parece bastante pertinente empezar el desarrollo de esta investigación con esta pregunta, ya que lo que se va a dar es una serie de reflexiones encadenadas: un parcheado de textos que, interpretados, interpelan y contextualizan. Así, este tejido textual tiene intención de dar pie a cuestionamientos sobre cuáles son las posibilidades de desestabilizar las estructuras que sustentan los patrones dominantes constituidos por el lenguaje.

Son muchas las interpretaciones que hay acerca de estos cuestionamientos y muchos los modos de realizar un enfrentamiento al problema epistemológico. Pueden encontrarse diversas posiciones dentro de la historia del arte, por una parte, queriendo operar fuera de los límites de la estructura de lenguaje a tratar, como la perspectiva en la que se encontraría Richard Serra, en su declaración de intenciones:

"Cada lenguaje tiene su propia estructura y no se puede criticar este si se utiliza el mismo lenguaje. Hace falta otro lenguaje, que trate de la estructura del primero y posea una estructura nueva, para criticarlo." (Serra, 2010: 171)

Existen también otras posiciones establecidas que interpretan las posibles brechas desde dentro, desestabilizando las estructuras en el mismo terreno desde el que se quiere transgredir. Estas reflexiones tratan de originar otros puntos de partida para llevar a cabo las permutaciones intencionadas desde las que se pretenden generar la ruptura. Lo que en palabras de Walter Benjamin se metaforizaría en la afirmación de que "solo mediante la poesía puede criticarse la poesía" (Azúa, 2011: 19).

En cualquier caso, esta problemática que se ubicaría en cualquier dirección y en cuales quieran que sean las prácticas que van a configurarse y a regirse a través de ella. Es en este punto en el que empezó a ser parte de un camino de reflexión filosófica y una toma de consciencia de la producción que se genera a través del lenguaje y los discursos. 


\section{Campos Gil, Isabel \\ El lenguaje y los movimientos de ocupación. Un recorrido por las nociones dominantes del lenguaje en relación con la ocupación como movimiento contra-cultural en la hegemonía occidental. \\ III CONGRESO INTERNACIONAL DE INVESTIGACIÓN EN ARTES VISUALES :: ANIAV 2017 :: GLOCAL [codificar, mediar, transformar, vivir] http://dx.doi.org/10.4995/ANIAV.2017.4892}

"El discurso no es simplemente aquello que traduce las luchas o los sistemas de dominación, sino aquello por lo que, y por medio de lo cual se lucha, aquel poder del que quiere uno adueñarse." (Foucault, 1992: 13)

Foucault ya elucubraba en El orden del discurso cómo podría enfrentarse al discurso desde el propio discurso; qué posibilidades de crítica existirían, criticando desde los mismos términos, y produciendo a través del lenguaje y de los discursos una crítica desde su propio terreno. Es decir, un cuestionamiento de cuál puede ser la potencialidad del discurso interrogando al discurso, interpelándolo.

Desde esta misma posición se encuentra Teresa de Lauretis. La autora trata de desafiar la teoría en sus propios términos, los términos de espacio semiótico construido en el lenguaje; una teoría tan bien establecidas que, paradójicamente, la única manera de situarse fuera de ese discurso es desplazarse dentro de él. Ejemplificado mucho más concretamente en la paradoja de la propia teoría feminista que es a la vez excluida del discurso, pero aprisionada en él (Lauretis, 1992:18)

\section{El lenguaje en la investigación y en las prácticas artísticas.}

En cuanto a las prácticas artísticas, las reflexiones epistemológicas del lenguaje han encadenado propuestas en las prácticas conceptuales durante un largo periodo, desde los años sesenta a los post-conceptualismos contemporáneos. Por ejemplo, la producción de Antoni Muntadas ha abarcado en su trayectoria artística el espacio público y sus metodologías de construcción, y así, en sus últimas producciones, ha puesto en cuestión las posibilidades de entender el lenguaje de ese mismo modo. De la misma manera que Muntadas en su exposición 'Palabras, palabras...' propuso una lectura sobre los significantes de palabras que han sufrido transformaciones a raíz de procesos de desgaste y de uso repetido por medio del poder político, se pueden reconocer ciertas tácticas que albergan las posibilidades de una metodología derivada de las transformaciones de mecanismos que provocan la deformatividad de los códigos lingüísticos, y que pueden invertirse hasta conseguir nuevas permutaciones.

En este caso podríamos hablar de una relación de reciprocidad del sujeto y su entorno, del sujeto y lo que le rodea, de un proceso sistémico influyente bilateralmente por ambas partes; lo que se podría definir como un proceso de reflexión del medio que habitamos, del medio que nos habita y de sus configurantes. Una red sistémica en la que cada elemento contiene un poder de actuación, de influencia, y que posee la capacidad de agencia. Entendiendo la construcción de la realidad desde una mirada posestructuralista, se puede llegar a ejercer ciertos puntos de permutación de los códigos a través de los cuales se pueden ir generando otros nuevos cambios, tal como diría Michael Craig Martin: precipitar el cambio.

Pero también me parecería importante, ya adentrados en la investigación semiótica y en relación a la construcción de realidades y construcciones culturales, cuestionar qué tipo de construcción significante produce la misma palabra construcción; Las acepciones lingüísticas que trabajan en el sustantivo de la palabra, pueden conllevar al significado constructo, el cual se habría creado para establecerse $y$, una vez establecido, formaría parte de cada individualidad, como sujeto sometido a este significante. Por lo que, esta concepción-acepción de la palabra construcción, conllevaría al abandono o a la pérdida de la capacidad de agencia que hace posible las acciones generadoras de realidades y la construcción de las mismas en un proceso de reciprocidad de producción.

Quizás es cierto que esta taxonomía pueda aproximarse a lo que en una primera lectura se pudiera entender como producción de subjetividades y acciones generadoras de contextos, pero puede que exista una disonancia entre lo que denota este término y los procesos en construcción de subjetividades.

\subsection{Sobre los espacios.}

La conversación sobre el espacio en el contexto del arte entre Barry, Huebler, Kosuth y Weiner, en el Simposio de 'Art Without Space' en Conceptual Art, de Peter Osborne, podemos obtener la diferenciación de Jeff Kelley que Lucy Lippard refleja en el texto Mirando alrededor: dónde estamos y donde deberíamos estar:

"El emplazamiento se refiere a las propiedades físicas que constituyen un lugar... mientras que los lugares son los recipientes de lo humano." (Kelley et al., 2011: 57)

Podríamos decir que este espacio interior, simbólico, es lo que da resultado al espacio como vertiente de lo humano; y de la misma manera, deberían considerarse procesos paralelos de producción. Es decir, lo que supondría un cambio en el funcionamiento semiótico-lingüístico, supondría un cambio en el espacio estructurado por esta articulación. Y, sirviéndose así de un proceso desestructural desde la epistemología del lenguaje como constructor, se situaría al lenguaje como principio y fundamento de esta gnoseología. 


\section{Campos Gil, Isabel \\ El lenguaje y los movimientos de ocupación. Un recorrido por las nociones dominantes del lenguaje en relación con la ocupación como movimiento contra-cultural en la hegemonía occidental. \\ III CONGRESO INTERNACIONAL DE INVESTIGACIÓN EN ARTES VISUALES :: ANIAV 2017 :: GLOCAL [codificar, mediar, transformar, vivir] http://dx.doi.org/10.4995/ANIAV.2017.4892}

En esta época, durante los seis años que analiza Lippard (2004), se desarrollaron numerosos trabajos como respuesta de un ataque al formalismo de los años anteriores. En este cultivo de subversión, se empieza a despojar de la idea del arte como experiencia estética para pasar a otros análisis de la experiencia.

“El arte dejó de consistir en un objeto para ser interpretado y paso a ser el centro de una investigación acerca del propio fenómeno del arte" (Morgan, 2013: 38)

En este contexto de toma de consciencia y participación en los mecanismos de la fenomenología del arte, los códigos culturales tomaron protagonismo en la investigación y formaron parte de los materiales del arte. Así, gran parte de las prácticas artísticas conceptuales tomaron el lenguaje como material artístico, como documento de significación y reflexión. Pasando del objeto artístico como fin, a la práctica como medio y herramienta (Morgan, 2013: 38-39).

Este momento de rechazo constituyó un terreno perfecto para la reflexión ante lo que había y ante lo que se quería generar, el tratado de las palabras como signos tuvo un gran papel en las prácticas conceptuales; a través del análisis del método estructuralista, que tiene como punto de partida la obra de Saussure, quien identifica el paradigma simbólico del lenguaje, que funcionaba a través de un sistema de organización, en el que sus elementos, el significante y el significado, se relacionan derivándose.

\subsection{Sobre la ocupación.}

En la estructuración formal semiótica, podríamos situarnos, ahora sí, dentro del espacio del ajedrez. Este estaría compuesto por un sistema en red de oposición binaria: los espacios a través de los que se constituiría el código del juego. En esta partida, los significados sausurrianos formarían parte de un conjunto más amplio o, en particular, se encontrarían sometidos a un principio o norma general que sería constituida por los signos, manteniendo una correlación permanente.

Siguiendo esta línea de Teresa de Lauretis (1992: 12), esta metáfora textual del ajedrez fue usada por lo que entenderíamos como la figura paterna del estructuralismo y, así, se ejerció la ley del padre en el espacio del orden simbólico organizado. Esta filosofía estructuralista del lenguaje, conformado como estructura y sistema, fue representada por el espacio del juego del ajedrez; el tablero y los códigos de la partida resultaban instruir lo que Saussure y Lévi-Strauss apoyaban como la única manera de poder entablar un intercambio utilizando como medida el lenguaje. Esto se vuelve un proceso problemático y conflictivo cuando se trata de sujetos que quieren desarticular y desarmar el sistema en el que se encuentran producidos y perpetuados por el propio lenguaje.

Realizando una lectura por las estrategias en los movimientos sociales, políticos y culturales de Occidente, y en relación con la resignificación de espacios, una de las posibilidades de actuación puede ser la adaptación de las estrategias de ocupación de espacios deshabitados. Ocupar, según el derrotero que nos interesa, significa apropiarse del terreno; lo cual, según este sentido, es interesante el desplazamiento terminológico y simbólico que puede permutarse de una estrategia que remite, a su vez, a una de las estrategias artísticas utilizadas por los movimientos feministas de las décadas de los 60 y 70.

La utilización de cualquiera de estos dos modos de apropiacionismo supone una propuesta de desvío en el entendimiento de un espacio en el que operan unos órdenes dominantes. A estos órdenes se les quiere otorgar una mirada distanciada y un desvío en el significado del espacio. Se trataría en última instancia, de habitar un espacio, de apropiarte de aquello que va a configurar a las individualidades y por lo que se quiere establecer un punto de ruptura en la lógica dominante.

En este punto sería consecuente analizar el terreno del juego del ajedrez en relación a los espacios físicos y semióticos que nos configuran. Existe un interés por el análisis del espacio de juego como régimen disciplinario y tanatopolítico, con el orden simbólico, organizado por el espacio, que sustentan las estructuras de la organización. Por un lado, la transferencia de planos -del real al juego-, evidencia como los órdenes dominantes que se van estableciendo en cada época no se extinguen a la llegada de la nueva adaptación a los nuevos paradigmas, si no que más bien se subsume uno dentro del otro, respondiendo a estructuras yuxtapuestas, estructura sobre estructura. 


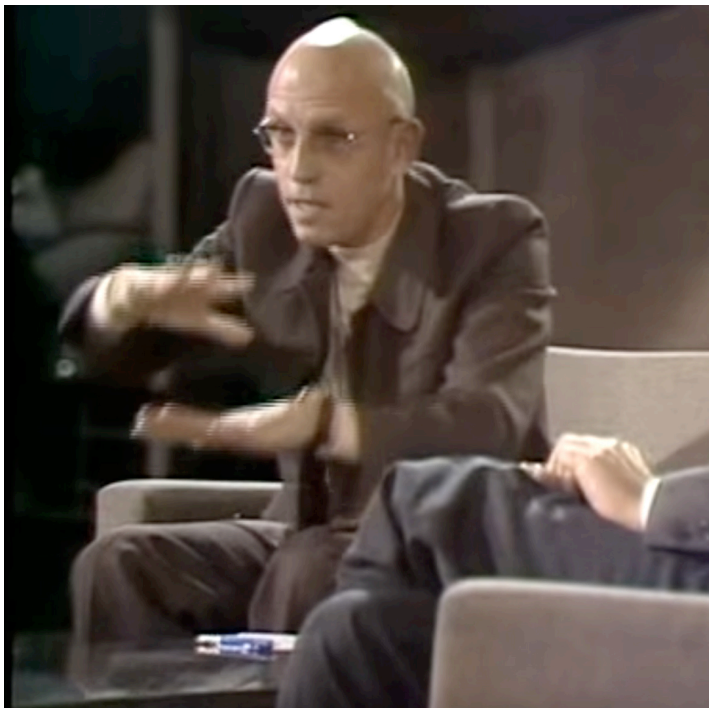

Imagen 1. Fotograma del debate entre Michael Foucault y Noam Chomsky en la Universidad de Amsterdam, 1971, dentro del International Philosophers Project.

En relación al análisis del espacio de ajedrez, con respecto al espacio por donde nos movemos como sujetos, el primer régimen que se puede evidenciar es el soberano -el cual se puede identificar como el orden dominante vigente hasta mediados del s. XVIII. Este tipo de dirección dio paso a un régimen mucho más característico para las sociedades posmodernas, un régimen que establece un poder sutil, según Foucault (2003: 39), "un poder discreto, repartido; es un poder que funciona en red y cuya visibilidad solo radica en la docilidad y la sumisión de aquellas personas sobre quienes se ejerce en silencio".

Podría ser pertinente visualizar desde un terreno tan cotidianamente iconificado y común como es el terreno del ajedrez, a ciertos elementos que puedan ser fácilmente transportados hacía el contexto en el que nos desenvolvemos. En esta superposición de regímenes y órdenes de dominantes se puede ver cómo, en cuanto a lo que al régimen disciplinario respecta, las técnicas de violencia, técnicas de vida y muerte no estorban a su funcionamiento. Estas técnicas son producidas y reproducidas por las masculinidades como formas de poder sobre los cuerpos subalternos.

Así como, a través del análisis del tipo de arquitectura panóptica ideada por Jeremy Bentham a finales del s. XVIII, Foucault (1975) estructura una de sus más populares investigaciones sobre cómo se consigue adaptar un régimen disciplinario a través de los espacios arquitectónicos, la arquitectura del espacio, como marco conceptual y estructural, genera los medios de interiorización y normalización de las prácticas corporales que se producen en ella. Desarrollándose así, una lectura sobre los espacios como estructuras sobre las que habitar una serie de conductas normativas, y que acaban convirtiéndose en las ficciones política que dan respuesta a los procesos de producción de subjetividades contemporáneas (Preciado, 2014).

Tal observación sobre como los espacios ejecutan como productores se hace evidente poco antes a la publicación del Vigilar y castigar de Foucault en la arquitectura producida por los movimientos de la izquierda marxista que empiezan a establecer una conciencia postmoderna en la que se hicieron coincidir en la crítica de la arquitectura del movimiento moderno una relectura a las disciplinas del modernismo industrial. En apenas una década, sobre el 1966 y 1974, marcada por las revueltas del 68 y las crisis del petróleo de 1973, diferentes grupos de la arquitectura europea se abren a las prácticas conceptuales, introduciéndose en ese estudio crítico la experiencialidad que se obtenía del contacto con una revolución marcada por los happenings, las instalaciones, el postcinema, los collages y las publicaciones fuera del marco normativo, pero también danza, poesía y eslóganes radicales como el "Todo es arquitectura" que sostiene Hans Hollein (1968) ${ }^{1}$.

\footnotetext{
${ }^{1}$ Esta reseña es una paráfrasis de la cartela referencial Los amigos de Fourier. El espacio es una producción social de la exposición: $1.000 \mathrm{~m} 2$ de deseo. Arquitectura y sexualidad, expuesta en el apartado temático: Refugios libertinos (s. XVIII-s. XX) Comisariada por: Adèlaïde de Carters y Rosa Ferré. CCCB, Centre de Cultura Contemporània de Barcelona. 25 de Octubre de 2016 - 19 de Marzo de 2017.
} 


\section{Conclusiones.}

En un proceso en construcción de una mirada post-critica desde los estudios queer, ocupar y habitar el lenguaje desde los sujetos subalternos supondría la re-significación del espacio de producción de las realidades. Esto tiene que ver con los procesos de producción de subjetividades, atendiendo a las construcciones desde una posición de transgresión a la normatividad. Si bien, es un proceso determinado de la relación con el contexto y la situación para establecer tales estrategias, y es que, de la misma manera que hoy se establecen reivindicaciones para existir dentro del orden del lenguaje, en otras épocas, por ejemplo las reconocidas por un régimen dictatorial totalitario, no encontrar el sitio en el orden del lenguaje dominante significaba una no existencia que conllevaría a no ser perseguido como sujeto abyecto.

En última instancia, dentro del contexto occidental contemporáneo estaríamos tratando de reflexionar sobre un proceso de ocupación del lenguaje como lugar de resistencia política, como estrategia de expropiación de las tecnologías y de producción de subjetividades. En este caso, sería necesario generar el contexto donde se puedan ejercer nuevas tácticas de invención de la libertad y producción de subjetividades, y para ello habría que funcionar desestabilizando la lógica dominante del juego. Tal y como recoge Teresa de Lauretis (1992: 12) del texto System and Structure de Anthony Wilden: "quienquiera que defina el código o el contexto tiene el control... y todas las respuestas que acepten ese contexto renuncian a la posibilidad de redefinirlo".

Fue en 1952 cuando David Tudor performativizó la composición 4’33 de John Cage, una pieza que supondría un desafío a la lógica y a la definición misma de una tautología disciplinaria que esperaba un funcionamiento o un comportamiento normativo. Esa permutación de los órdenes que operaban dentro de la disciplina y las composiciones musicales, pudo no ser entendida por una gran parte de público, incluso pudo ser injuriada desde una perspectiva formalista sobre que era arte y que no. Pero fundamentalmente marcó una diferencia y marcó las posibilidades en el momento de inicio de los ejercicios de inversión de la lógica normativa estructural.

¿Qué pasaría si nos encontramos en una partida de ajedrez, con un primer movimiento de blancas, y en nuestro puesto esperamos 4'33 y nos ausentamos? ¿Qué pasaría si el caballo quiere ser la tuerca del urinario de Duchamp y quiere moverse como la reina?

"Considera aún este caso. • Le explico a alguien el ajedrez; y comienzo señalando una pieza y diciendo: «Éste es el rey. Puede moverse así y así, etc., etc.». - En este caso diremos: las palabras "Este es el rey» (o "Esta se llama 'rey'») son una explicación de la palabra sólo si el aprendiz ya 'sabe lo que es una pieza de un juego'. Es decir, si ya ha jugado otros juegos o ha observado 'con comprensión' el juego de otros - y cosas similares. Sólo entonces podrá también preguntar relevantemente al aprender el juego: «¿Cómo se llama esto?»

Podemos decir: Sólo pregunta con sentido por la denominación quien ya sabe servirse de ella.

Podemos también imaginarnos que el interrogado responde: "Decide la denominación tú mismo» - y ahora el que ha preguntado debe responder de todo por sí mismo." (Wittgenstein, 1958: 18-19)

\section{FUENTES REFERENCIALES.}

DE AZUA, F. 2011. Diccionario de las artes. Madrid: DEBATE. ISBN 9788499920030.

DE CARTERS, A. FERRÉ, R. (com.). $1.000 \mathrm{~m} 2$ de deseo. Arquitectura y Sexualidad. (Exposición celebrada en Barcelona, CCCB, del 25-X2016 al 19-III-2017). Barcelona, CCCB, 2017.

DE LAURETIS, T. 1992. Alicia ya no. Madrid: Ediciones Cátedra, S.A. ISBN 8437611407

FOUCAULT, M. 1999. El orden del discurso. Buenos Aires: Tusquets Editores. ISBN 9788483106549.

FOUCAULT, M. 2003. El poder psiquiátrico. Madrid: Akal. ISBN 9788446021100

KRISTEVA, J. 1999. El lenguaje, ese desconocido: Introducción a la lingüística. Madrid: Ediciones Fundamentos. ISBN 9788424504984. 
Campos Gil, Isabel

El lenguaje y los movimientos de ocupación. Un recorrido por las nociones dominantes del lenguaje en relación con la ocupación como movimiento contra-cultural en la hegemonía occidental.

III CONGRESO INTERNACIONAL DE INVESTIGACIÓN EN ARTES VISUALES :: ANIAV 2017 :: GLOCAL [codificar, mediar, transformar, vivir] http://dx.doi.org/10.4995/ANIAV.2017.4892

MORGAN, R. C. 2013. Del arte a la idea. Madrid: Akal. ISBN 9788446011644.

PRECIADO, P. B. 2014. "Las subjetividades como ficciones políticas". Video Youtube. Obtenido de: https://youtu.be/R4GnRZ7 -w4 Consultado: 04/02/2017.

V.V.A.A. 2001. Mirando alrededor: dónde estamos y donde deberíamos estar. En Modos de hacer: Arte crítico, esfera pública y acción directa. Salamanca: Ediciones Universidad de Salamanca. ISBN 9788478008926

V.V.A.A. 2011. Richard Serra: Escritos y entrevistas 1972-2008. Pamplona: Universidad Pública de Navarra. ISBN 9788497692625.

WITTGENSTEIN, L. 1999. Investigaciones filosóficas. Madrid: Ediciones Atalaya, S.A. ISBN: 8448712501. 